\title{
DATABASE FINLIV - FOCUS ON STAIRCASE METHOD
}

\author{
Maxim Lutovinov*, Jan Papuga, Milan RŮŽIČKa \\ Faculty of Mechanical Engineering of Czech Technical University in Prague, Technická 4, Prague, Czech \\ Republic \\ * corresponding author: maxim.lutovinov@fs.cvut.cz
}

\begin{abstract}
This paper introduces FinLiv database focused on gathering, manipulating and providing experimental static and fatigue data. One of its new features is the ability to include also fatigue tests realized by the staircase method.
\end{abstract}

KEYWORDS: fatigue, database, staircase method, fatigue limit.

\section{INTRODUCTION}

FinLiv is a database intended to gather the information about static and stress- and strain-controlled fatigue experiments and also about parameters of various static and fatigue material models derived from such data. It was developed to resolve the problems of researchers in a lack of quality data, their frequent second-hand use, and the way the data items are selected for various benchmark tests. By now, FinLiv has a large set of tools for entering and processing data. One of the newest tools is the staircase method [1] that automatically evaluates data from stress-controlled experiments and provides the fatigue limit as a result.

\section{FinLiv}

FinLiv is represented by two parts, which closely cooperate, but which also can be used as standalone applications. The first part serves for entering data, while the second part serves for listing them. The second part is implemented as a web database and can be found at www.fadoff.cz/page/finliv. It allows the user to have permanent access to the raw experimental data or to the final regression curves retrieved from them. In the web part there are no tools to process the data, it simply retrieves and lists them on a request in a raw or processed form.

The first part, FinLiv.VBA 2, was built as an MS Excel application written in Visual Basic for Applications, and its main purpose is to enter experimental data and to process them. Data processing is necessary in order to e.g. perform regression analysis or to prepare automated input for fatigue solvers, if a particular experimental data set has to be used within a benchmark of a particular calculation method. The MS Excel platform was chosen because of its prevalence among engineers, and because it is substantially cheaper than Matlab. The application can be accessed from any computer which has MS Excel installed.

The main interface of FinLiv.VBA is represented by "form" sheet (see the part of the input interface in Figure 11. It consists of a group of tables, combo boxes and buttons. Data input is realized by filling in the salmon colored cells and by choosing options from the combo boxes. In the first non-empty column there are designations that correspond to the input parameters. The same designations are used in the datasheets (Figure 2), where the material data are stored. The datasheets are placed in the same workbook as the "form" sheet, and their structure is quite simple: in the second column there are designations that represent the entered parameters, while the other columns contain information on individual curves of the particular data set.

FinLiv works with data from static experiments and stress- and strain-controlled fatigue experiments. Since each type of experiments uses a different set of parameters, the "form" sheet changes its structure to accommodate the selected type.

In addition to entering data, FinLiv.VBA can also process data. With the help of the application the user can easily perform regression analyses of experimental data and obtain corresponding regression parameters. For strain-life data there are two types of a regression analysis. The first one is carried out in a conventional way, which is represented by linear regression using the least square method. The second method performs non-linear regression according to the $3-\mathrm{D}$ method 3 . The 3-D method is based on the assumption that one value of the total strain amplitude corresponds to only one stress amplitude and to a single number of cycles. If this assumption is valid a curve can be drawn in a 3-dimensional space which besides formulation of the total characteristics depicts also plastic and elastic strain. Unfortunately, implementing such a non-linear $3-\mathrm{D}$ regression in Excel is problematic, therefore it was decided to carry out the regression analyses in an external Matlab application. To prepare an input file for that application the user should click "Input for Regression" button. To load the resulting parameters of the regression analysis to FinLiv, the user should click "Get regression data" button and choose the output file created by the Matlab application.

For stress-life data, there are also two types of a regression analysis, but unlike the case of strain-life 

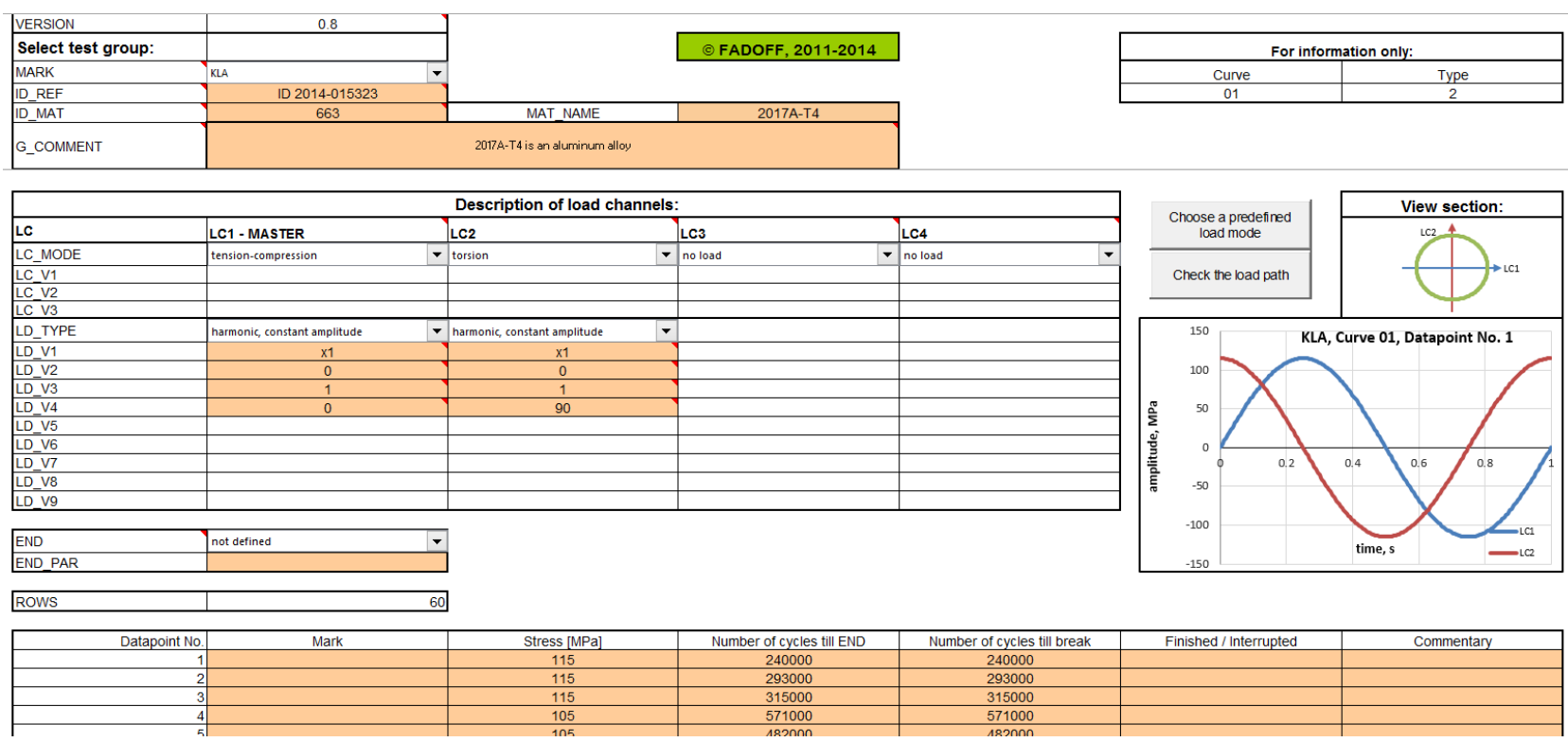

Figure 1. "Form" sheet of FinLiv with sample data.

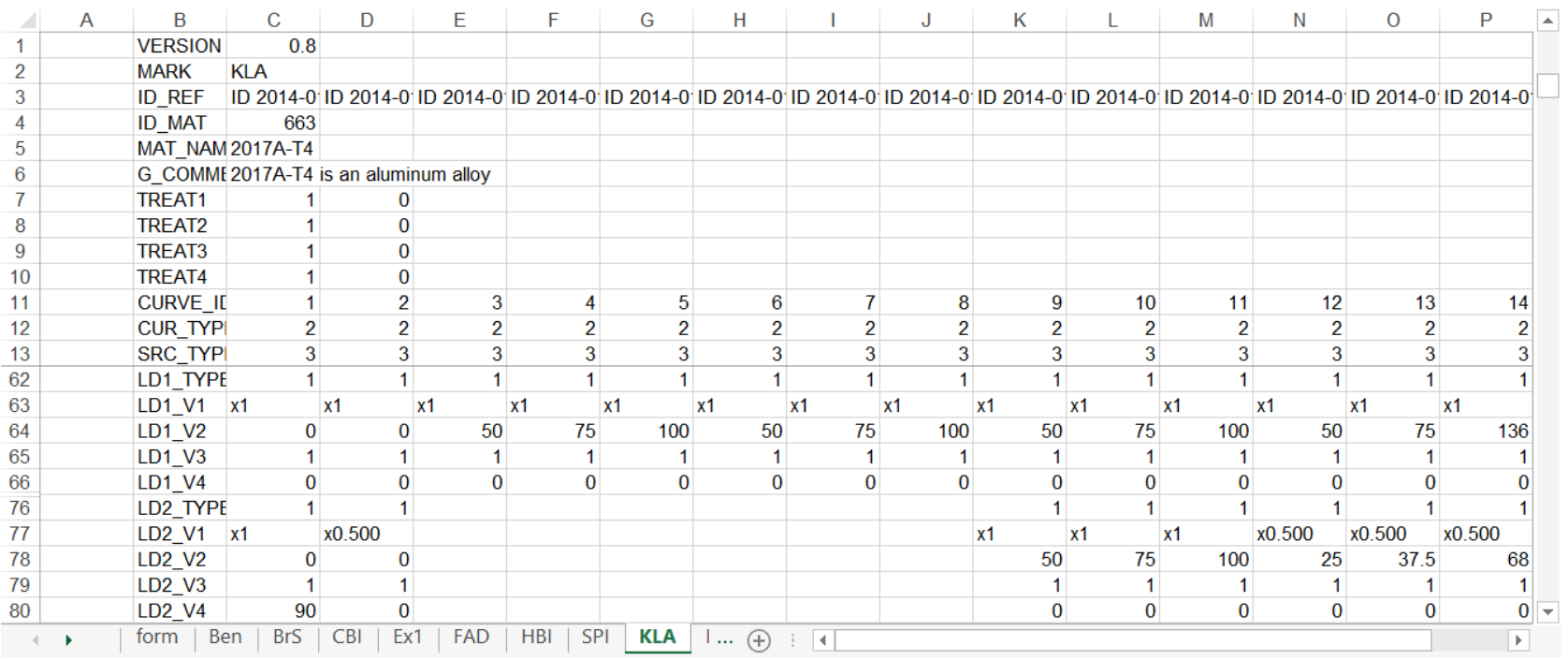

Figure 2. A datasheet of FinLiv with sample data.

data both types of the regression are carried out in the Excel environment. Regression analyses of S-N data as well as a visual display of obtained e-N curves are available in another workbook that is generated automatically from FinLiv. Individual sheets in that workbook correspond to the data sets that were selected for processing. At each page of such data there are two plots of regression curves. In the case of strain-life data, there are two Manson-Coffin curves, constructed on the basis of parameters obtained from conventional regression analyses and 3-D analyses. In the case of stress-life data, the first regression curve is represented by the Basquin relation and another one by Kohout-Vechet relation. For S-N data, a new tool is also available in the same workbook - the staircase method, which is described below.

\subsection{Staircase Method}

The staircase method is a standardized method [1 for carrying out and evaluating experiments in order to determine the fatigue limit related to a given number of cycles. The essence of the method is to increase or decrease the load amplitude of a new test specimen by a constant pre-selected value based on the response of the previous specimen, i.e. whether a failure occurred during the previous experiment.

For evaluation, the staircase method uses a special table (Figure 3) constructed according to the standard ISO 3800 [1]. The rows of the table represent stress levels and the columns represent the order of the experiments. A symbol "o" is entered into the appropriate cell when a failure doesn't occur, and a symbol " $x$ " in the opposite case. If the essence of the method has been respected, then the symbols " $\mathrm{x}$ " and "o" are arranged in the table into the sequence that 


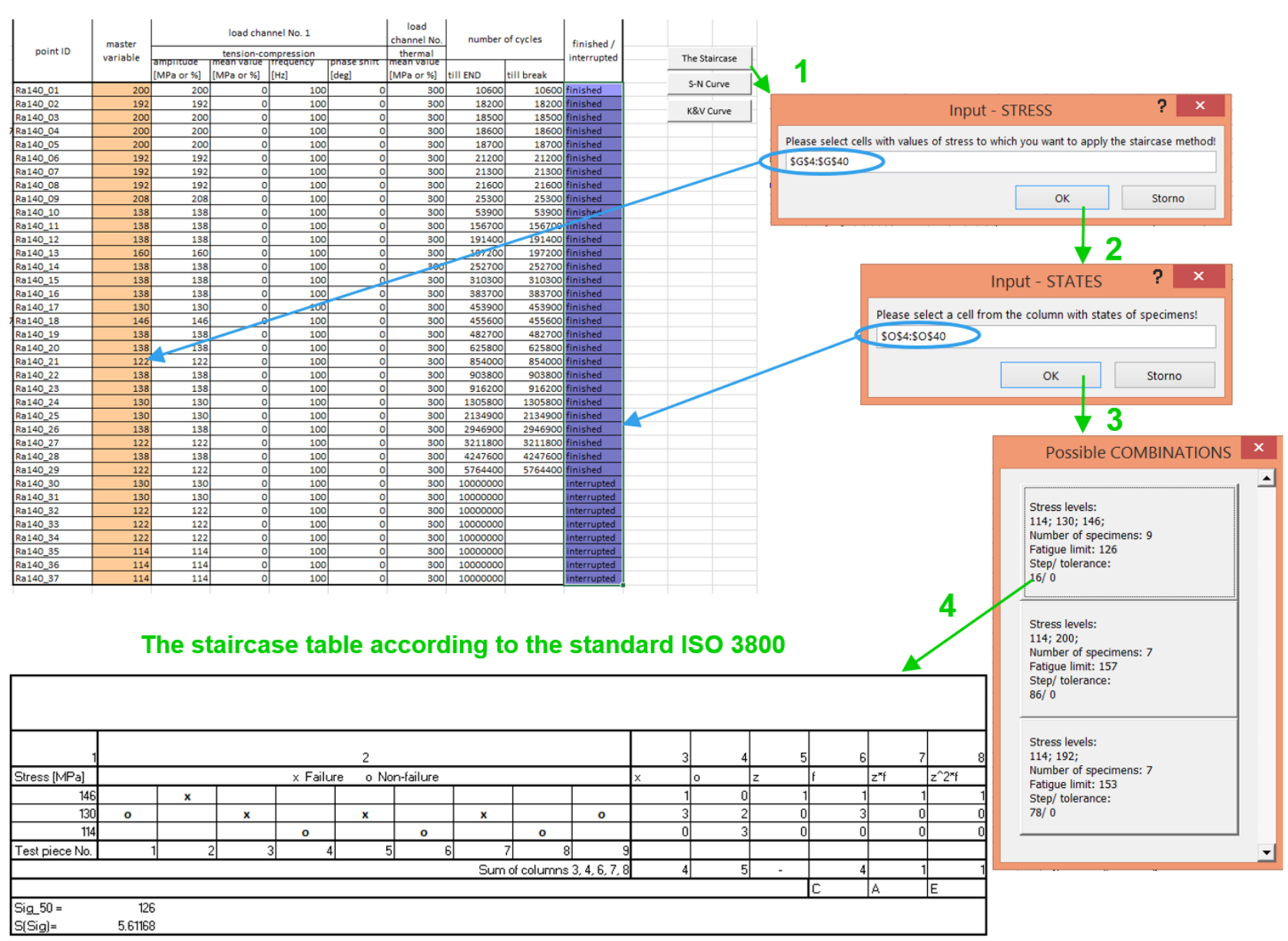

Figure 3. Process of generating the output table according to the staircase method in FinLiv.

looks like stairs. In case of a suitable choice of the first stress level and a step size between individual levels, the stress levels after several experiments (about 10 experiments) will oscillate around a certain value - the fatigue limit. The fatigue limit and the corresponding standard deviation can be then calculated with the help of the equations given by the standard [1, which uses values from the left part of the table (Figure 3 . columns 3 to 8). The fatigue limit labeled as Sig_50 in Figure 3 is calculated according to:

$$
S i g \_50=\sigma_{\text {ao }}+\Delta \sigma_{\text {all }}\left(\frac{A}{C}+x\right)
$$

Here, $\sigma_{a o}$ is the lowest load amplitude level at which the first non-failed specimen appears, given that the total sum of the non-failed specimens is less than the total sum of the failed specimens. In the opposite case, the load level corresponding to the first failed specimen should be input. The standard doesn't specify how to proceed in the case when the total sums of the failed and non-failed specimens are equal. The observations show that in such case the result will be the same regardless of whether failed or non-failed specimens are selected as the reference type. The algorithm implemented in FinLiv in cases like that calculates the fatigue limit in two steps, with the failed and non-failed specimens as the reference type and then chooses the lowest fatigue limit.

The parameter $\Delta \sigma_{\text {all }}$ is the step size between individual load levels. $A$ and $C$ are sums of the values in the columns $z^{*} f$ and $f$. The parameter $x$ is equal to +0.5 when the number of the non-failed specimens is lower than the number of the failed specimens, or $-0,5$ in the opposite case. It is worth noting that the method can be applied to the load levels represented by stress as well as to the levels represented by forces. The result is then the fatigue limit or the force corresponding to the fatigue limit.

The parameter $S(\mathrm{Sig})$ is a standard deviation of the distribution and is calculated according to:

$$
S(S i g)=1.62 \Delta \sigma_{\text {all }}\left(\frac{C E-A_{2}}{C_{2}}+0.029\right)
$$

where $E$ is the sum of values in the column $z^{\wedge} 2^{*} f$.

The staircase method was extended in FinLiv, and the application analyzes whether the data are suitable for being processed by the staircase method at all. Thanks to that, the method can also be used on data that were obtained from experiments initially unintended to be processed by the staircase method. If the data are suitable for the method, FinLiv automatically draws an output table (Figure 3).

The algorithm that analyzes data from the selected load levels assembles various combinations that differ 
in the step size between levels and/or by the number of levels. Then individual combinations are checked according to the criteria that say whether individual sets of load levels and corresponding sets of specimen states (failure, non-failure) are suitable for evaluation by the staircase method. These criteria are derived from the essence of the method. For example, one of the criteria says that if the number of the non-failed specimens at the highest load level is more than one, then such set of load levels and specimen states is not suitable for the staircase method. According to the method, the appearance of a non-failed specimen leads to increasing the value of load amplitude. If the experiment with such specimen is not the last one, then the next experiment should be carried out at a higher level of load amplitude, which means that the level of load amplitude corresponding to the non-failed specimen in question is not the highest level in the particular set of experiments. In the other case, if the experiment at the highest load level with non-failed is the last one, then at the same level there cannot be other non-failed specimens.

After selected data are checked by the criteria, FinLiv provides the user the list of possible combinations of load levels and corresponding steps to choose from (Figure 3). The combinations are ordered by the number of included specimens. For each combination there are also listed corresponding step sizes and calculated fatigue limits. While selecting a combination it is important to check the ratio of the fatigue limit and the step size. The smaller is the ratio, the more accurate the estimate should be.
The drawback of the implemented check is that it works only with load levels and can't take into account the possibility of individual specimens being removed from evaluation in order to assemble a suitable combination of the load levels and specimen states.

\section{Summary}

The paper refers to FinLiv database for storing, listing and manipulating experimental static and fatigue data. It describes its newest feature, which is the ability to process data by the staircase method.

\section{ACKNOWLEDGEMENTS}

The authors thank for the support of their research by SGS14/181/OHK2/3T/12 CTU grant.

\section{REFERENCES}

[1] ISO 3800:1993(E). Threaded fasteners - Axial load fatigue testing - Test methods and evaluation of results. Switzerland: International Organization for Standardization, 1993.

[2] J. Papuga, M. Lutovinov. Help for FinLiv.VBA Excel database [ver. C], FME CTU in Prague \& Evektor, spol. s.r.o., Prague 2014.

[3] A. Nieslony, C. Dsoki, H. Kaufmann, P. Krug. New method for evaluation of the Manson-Coffin-Basquin and Ramberg-Osgood equations with respect to compatibility. International Journal of Fatigue 30(1011):1967-1977, 2008. DOI:10.1016/j.ijfatigue.2008.01.012. 